\title{
Proceeding
}

7th INSHS International Christmas Sport Scientific Conference, 9-12 December 2012. International Network of Sport and Health Science. Szombathely, Hungary

\section{Exercise intensity during Zumba fitness and Tae-bo aerobics}

\author{
KRISTÍNA HIŽNAYOVÁ
}

Faculty of Physical Education and Sport, Comenius University Bratislava, Slovakia

\begin{abstract}
Hižnayová K. Exercise intensity during Zumba fitness and Tae-bo aerobics. J. Hum. Sport Exerc. Vol.8, No. Proc2, pp. S228-S241, 2013. This study analyzed and compared the exercise intensity in Zumba fitness and Tae-bo aerobics. Object of the research was monitoring of the heart rate and energy consumption in kcal during exercise unit, by using Heart Rate Monitor Suunto Memory Belt. All measurements were attended by randomly selected 11 women. The data we collected during the six units of the Zumba fitness and six units of the Tae-bo aerobics. Obtained data of the heart rate were evaluated in three main parts of workout: after warm up, in the main part of the workout and after the cool-down and final stretch. Energy consumption in kcal was evaluated after finishing exercise unit. Median of the heart rates prove, that the exercise intensity in the warm up and in the main part of workout was significantly higher in all 6 zumba fitness units than in tae-bo aerobics $(p<0.05)$. Documented energy consumption (in kcal) burned per whole workout was also significantly higher $(p<0.01)$ in all 6 Zumba fitness units. The research was conducted with the support of VEGA, grant project designed in the years 2011 - 2013, no. 1/0503/11 entitled: Functional and motor skills of athletes of all ages and performance in relation to selected parameters of the cardiovascular system. Key words: ZUMBA FITNESS, TAE-BO AEROBICS, EXERCISE INTENSITY, HEART RATE, ENERGY CONSUMPTION.
\end{abstract}

Corresponding author. Nábrežie arm. gen. L. Svobodu 9, 814 69, Bratislava, Slovakia

E-mail: hiznayova@fsport.uniba.sk

7th INSHS International Christmas Sport Scientific Conference, 9-12 December 2012. International Network of Sport and Health Science. Szombathely, Hungary.

JOURNAL OF HUMAN SPORT \& EXERCISE ISSN 1988-5202

(c) Faculty of Education. University of Alicante

doi:10.4100/jhse.2012.8.Proc2.26 


\section{INTRODUCTION}

The objective of the research was to compare the physiological load in zumba fitness and tae-bo aerobics, two popular types of group exercise, by monitoring of the heart rate and energy consumption in kcal during exercise unit.

Zumba fitness is a new kind of dance workout, inspired by Latin American music and Latin American dances. This exercise combines the basics of dance merengue, salsa, samba, cumbia, reggaeton and other Latin American dances, uses basic aerobic steps, but also enriches their composition of the other dances like hip-hop, belly dancing, Indian, African dance, etc. (Perez and Greenwood-Robinson, 2009). Greer (2000) said that Tae Bo is a form of high impact aerobics that combines the moves of Tae Kwon do, karate, boxing, ballet and hip-hop dancing. Tae Bo, which stands for Total Awareness Excellent Body Obedience was developed by Billy Blanks in the late 1980's.

Optimal Intensity of the recreational training can be expressed by the (Hrčka, 2000):

- Percentage of maximum heart rate (usually in the range of 60 to $90 \%$ ),

- Values of energy consumption (expressed in kcal kj and MTE s),

- Values of maximum oxygen consumption (VO2max).

\section{MATERIAL AND METHODS}

\section{Participants}

All measurements were attended by randomly selected 11 women aged 19 to 35 years (Table 1).

Table 1. The basic characteristics of the participants

\begin{tabular}{ccc}
\hline & Age & BMI \\
\hline Average & 26.57143 & 19.67273 \\
Stdev & 6.187545 & 0.757748 \\
Median & 25 & 19.7 \\
Min & 19 & 18.8 \\
Max & 35 & 21.2 \\
Variance & 16 & 2.4 \\
\hline
\end{tabular}

\section{Methodology}

Data were obtained by using Heart Rate Monitor Suunto Memory Belt. Data we collected during the six units of the Zumba fitness and six units of the Tae-bo aerobics. Each unit took 60 minutes. Heart rates were evaluated in three main parts of workout: after the warm up, in the main part of the workout (average of 3 values - in the 15th minute, 30th minute and 45th minute) and after final stretch (at the end of the lesson). Each exercise was led by another instructor. Instructors had to adhere assigned time limit, so in all measurements warm-up took 10 minutes, main part took 45 minutes, and cool down and final stretch took 5 minutes. Energy consumption in kcal was evaluated after finishing exercise unit. 


\section{Data analysis}

Physiological heart rate curves were processed by computer software data processing system. For comparison of data we used mathematical and statistical methods. To calculate the dependence between the values we used Wilcoxon T-test.

\section{RESULTS}

Middle values of the heart rate measurements obtained in 6 Zumba fitness classes (Figure 1) and in 6 Taebo aerobics classes (Figure 2).

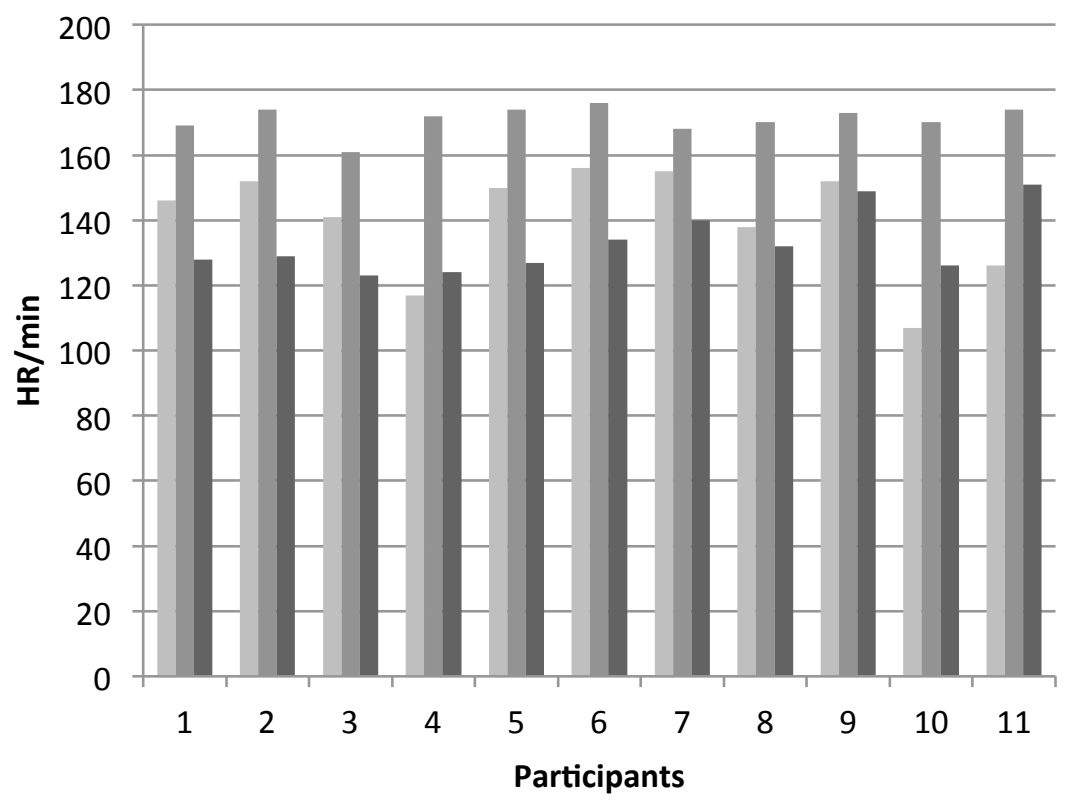

HR after warm up

HR in main part

HR after final stretch

Figure 1. Median HR in Zumba fitness

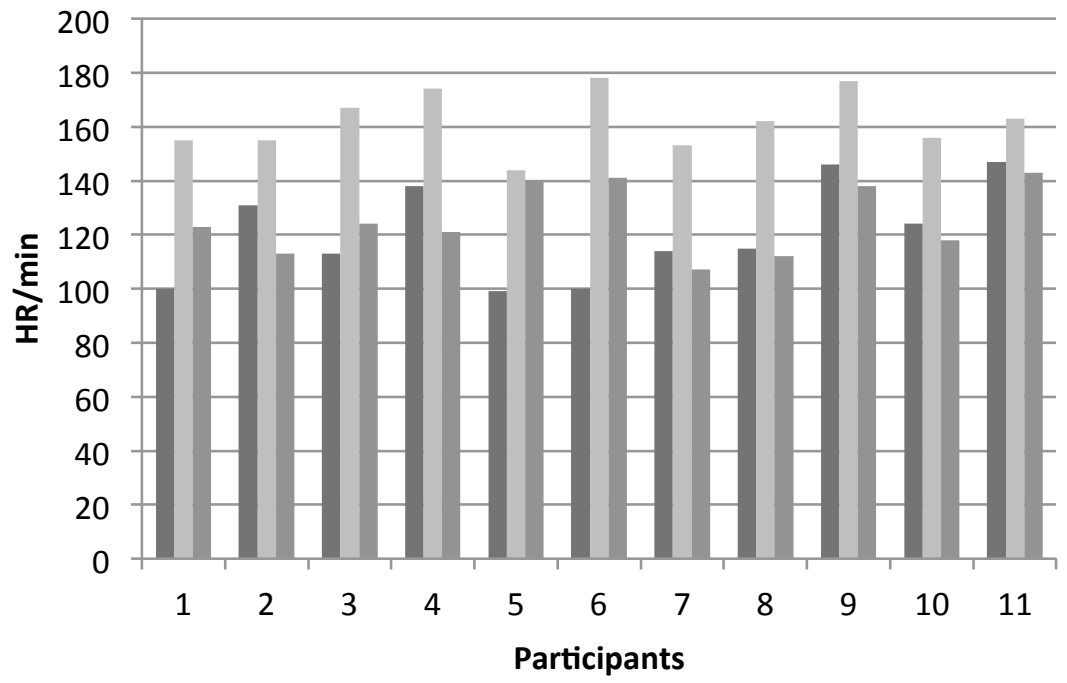

HR after warm up

HR in main part

HR after final stretch

Figure 2. Median HR in Tae-bo aerobics 
Median values of heart rate in main part of 6 Zumba fitness classes were in the zone $80 \%$ to $90 \%$ of individual maximum heart rate of participants (only one participant was above the $90 \%$ of maximum heart rate). Median values of heart rate in main part of 6 Tae-bo aerobics classes were by $63 \%$ of participants in the zone $80 \%$ to $90 \%$ of individual maximum heart rate. Three participants had median values of heart rate in main part of Tae-bo aerobics classes in zone $74 \%$ to $79 \%$ of maximum heart rate. One participant exceeded the anaerobic threshold ( $94 \%$ of maximum heart rate). It was a 31-year old participant who had the highest value of BMI among all (21.2). But such a value is considered as a normal, so we assume that higher values of heart rate caused mild disease, which broke participant at the time of measurements. Individual aerobic zone and curve of median values of heart rate in Zumba fitness and Tae-bo aerobics shown in Figures 3-24.

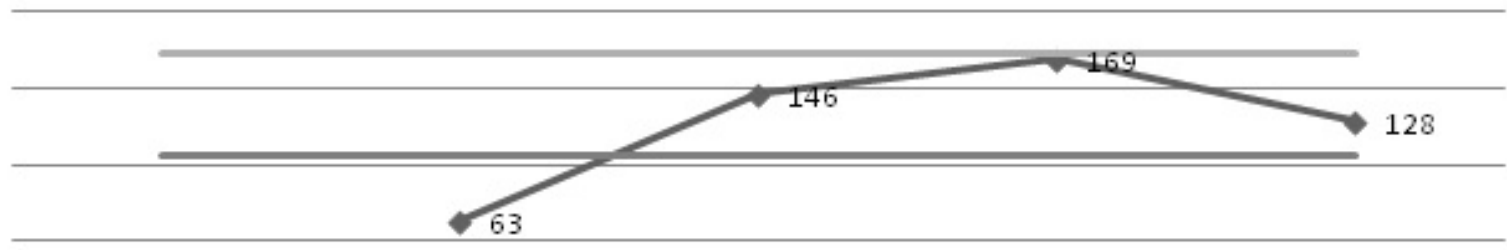

$\longrightarrow$ Heartrate $-90 \% \max \mathrm{HR} \longrightarrow 55 \% \max \mathrm{HR}$

Figure 3. Participant $n .1$ - curve of median values of heart rate in Zumba fitness

Notes: 63 - resting heart rate, 146 - heart rate after warm up, 169 - average heart rate in main part, 128 heart rate after final stretching.

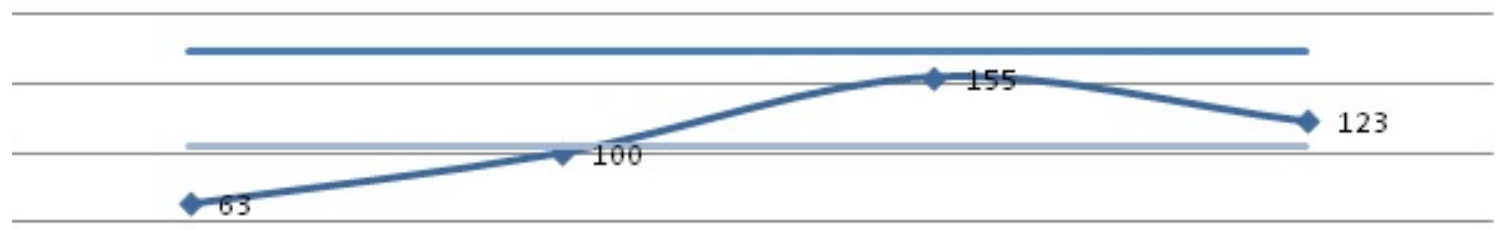

$\longrightarrow$ Heartrate $-90 \% \max \mathrm{HR}-55 \% \max \mathrm{HR}$

Figure 4. Participant $n .1$ - curve of median values of heart rate in Tae-bo aerobics

Notes: 63 - resting heart rate, 100 - heart rate after warm up, 155 - average heart rate in main part, 123 heart rate after final stretching. 

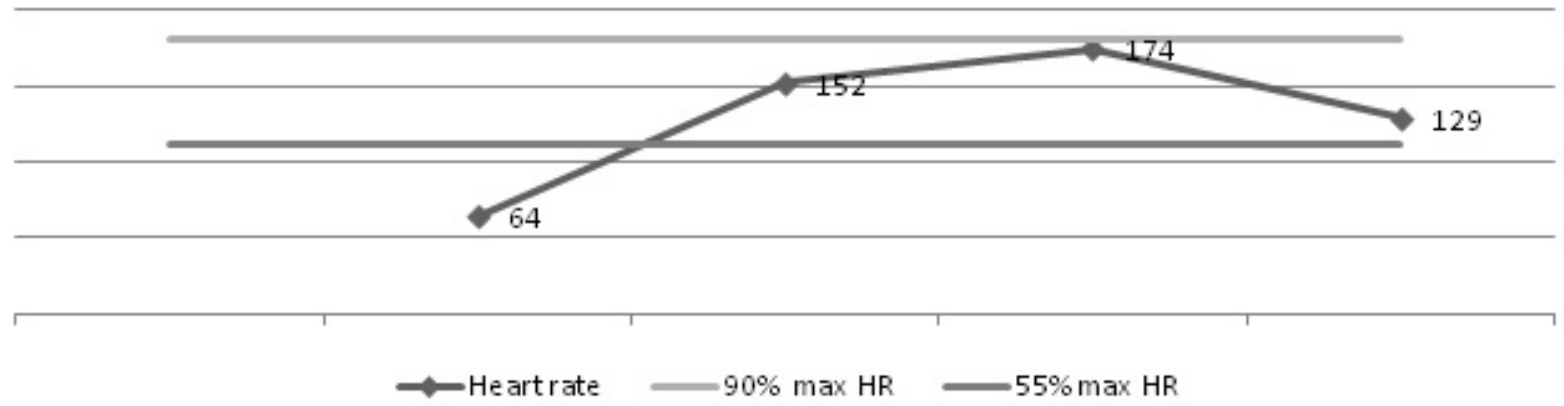

Figure 5. Participant n. 2 - curve of median values of heart rate in Zumba fitness

Notes: 64 - resting heart rate, 152 - heart rate after warm up, 174 - average heart rate in main part, 129 heart rate after final stretching.

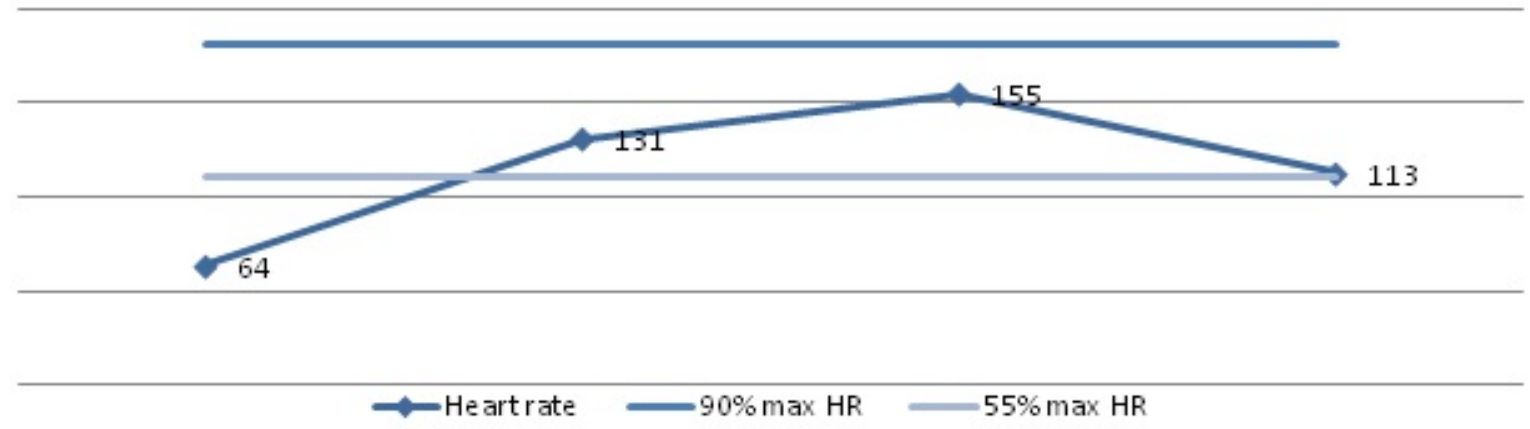

Figure 6. Participant n. 2 - curve of median values of heart rate in Tae-bo aerobics

Notes: 64 - resting heart rate, 131- heart rate after warm up, 155 - average heart rate in main part, 113 heart rate after final stretching.

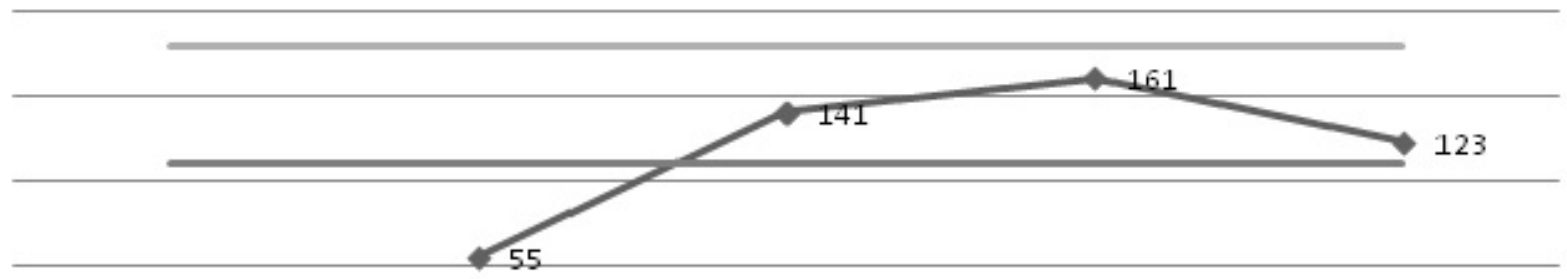

$\longrightarrow$ Heartrate $-90 \% \operatorname{max~HR}-55 \% \max \mathrm{HR}$

Figure 7. Participant n. 3 - curve of median values of heart rate in Zumba fitness

Notes: 55 - resting heart rate, 141- heart rate after warm up, 161 - average heart rate in main part. 


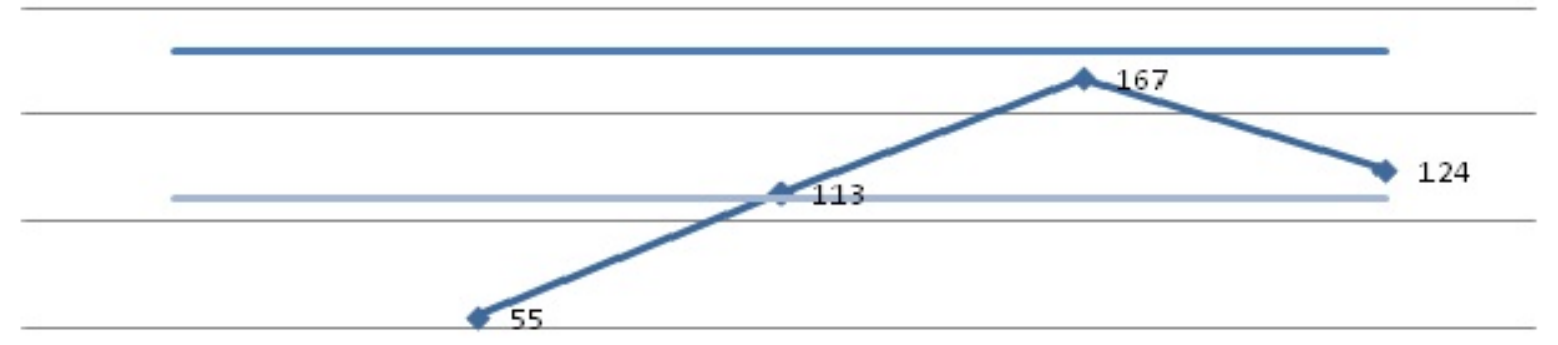

$$
\text { - Heart rate }-90 \% \operatorname{max~HR}=55 \% \max \mathrm{HR}
$$

Figure 8. Participant n. 3 - curve of median values of heart rate in Tae-bo aerobics

Notes: 55 - resting heart rate, 113- heart rate after warm up, 167 - average heart rate in main part, 124 heart rate after final stretching.

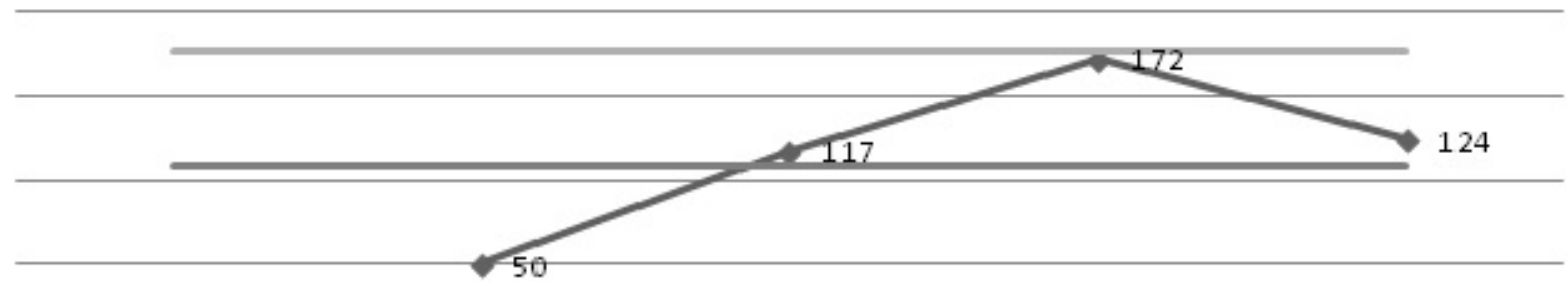

- Heartrate $-90 \% \max \mathrm{HR}=55 \% \max \mathrm{HR}$

Figure 9. Participant n. 4 - curve of median values of heart rate in Zumba fitness

Notes: 50 - resting heart rate, 117- heart rate after warm up, 172 - average heart rate in main part, 124 heart rate after final stretching.

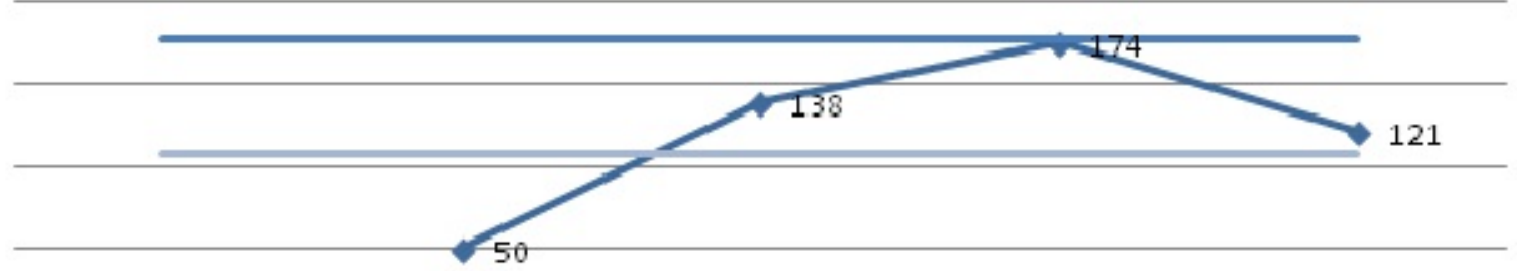

$$
- \text { Heartrate }-90 \% \max H R \quad 55 \% \max H R
$$

Figure 10. Participant n. 4 - curve of median values of heart rate in Tae-bo aerobics

Notes: 50 - resting heart rate, 138- heart rate after warm up, 174 - average heart rate in main part, 121 heart rate after final stretching. 


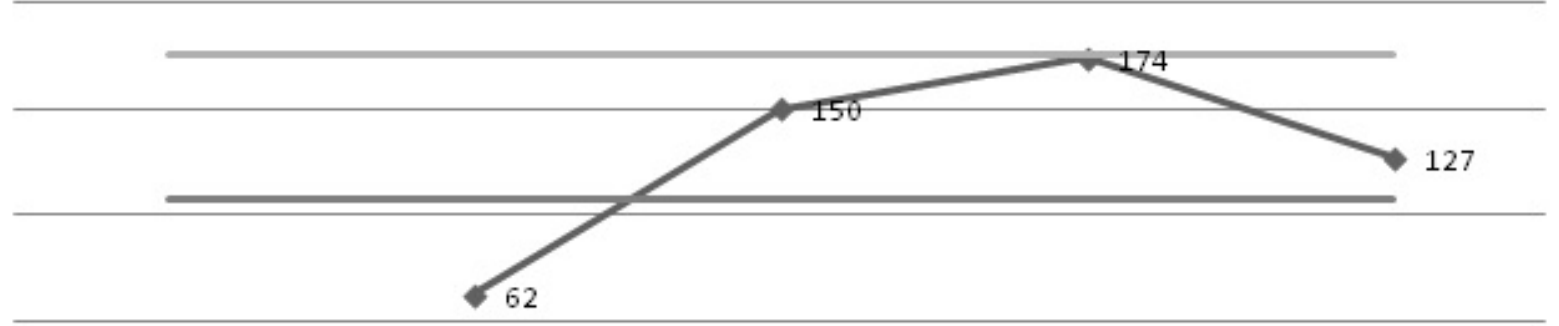

- Heartrate $-90 \% \max \mathrm{HR}=55 \% \max \mathrm{HR}$

Figure 11. Participant $n .5$ - curve of median values of heart rate in Zumba fitness

Notes: 62 - resting heart rate, 150- heart rate after warm up, 174 - average heart rate in main part, 127 heart rate after final stretching.

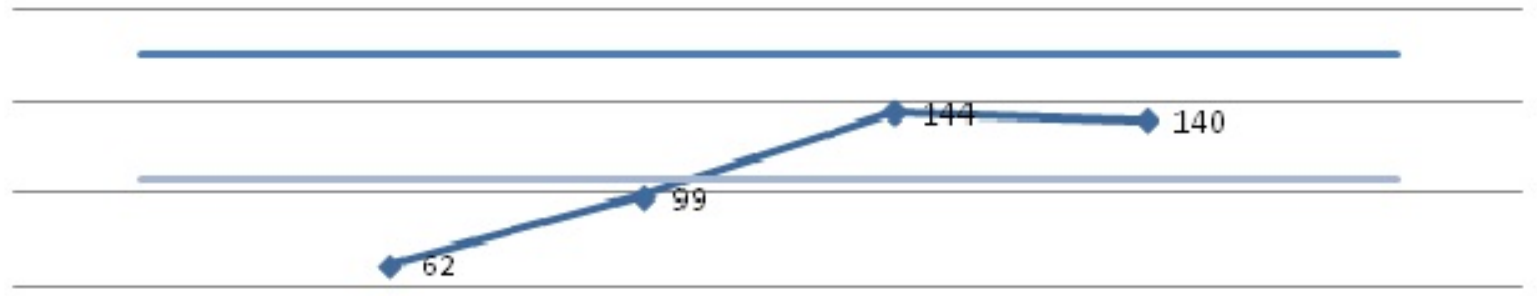

$=$ He artrate $=90 \% \operatorname{max~HR}=55 \% \operatorname{max~HR}$

Figure 12. Participant $n .5$ - curve of median values of heart rate in Tae-bo aerobics

Notes: 62 - resting heart rate, 99- heart rate after warm up, 144 - average heart rate in main part, 140 heart rate after final stretching.

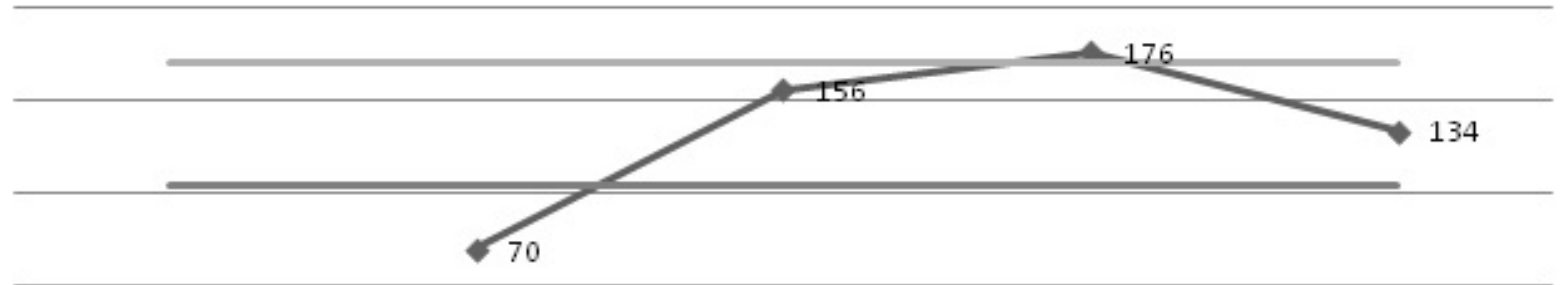

- Heartrate $-90 \% \max \mathrm{HR}=55 \% \max \mathrm{HR}$

Figure 13. Participant $n$. 6 - curve of median values of heart rate in Zumba fitness

Notes: 70 - resting heart rate, 156 - heart rate after warm up, 176 - average heart rate in main part, 134heart rate after final stretching. 


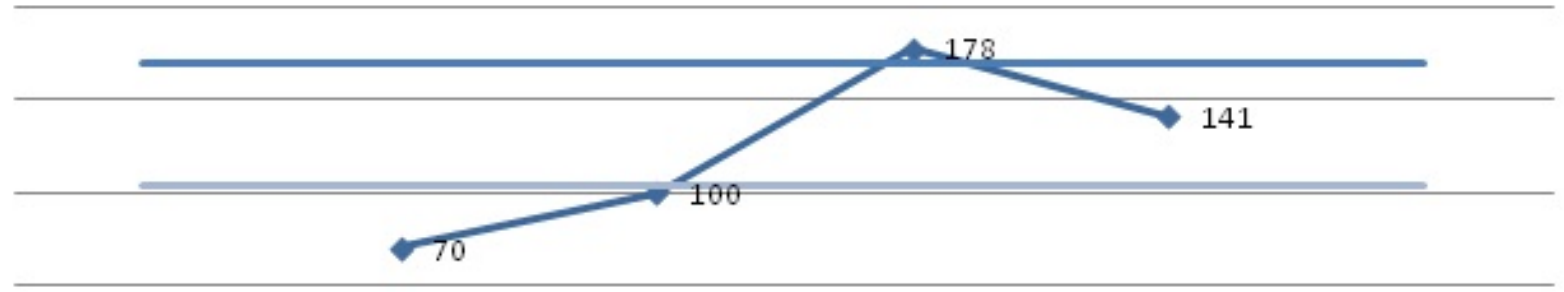

- Heartrate $-90 \% \max \mathrm{HR}=55 \% \max \mathrm{HR}$

Figure 14. Participant $n .6$ - curve of median values of heart rate in Tae-bo aerobics

Notes: 70 - resting heart rate, 100- heart rate after warm up, 178 - average heart rate in main part, 141heart rate after final stretching.

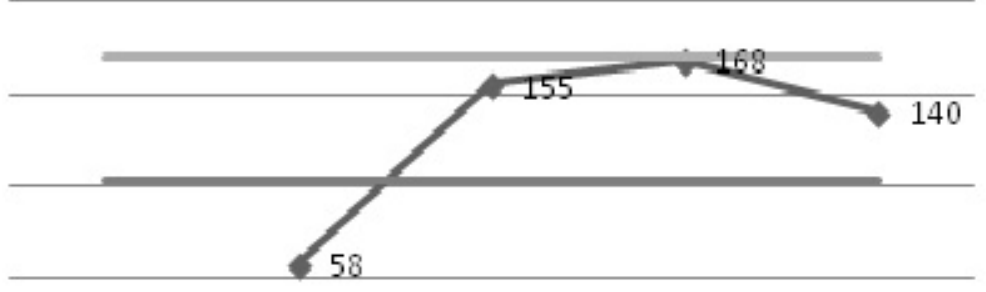

- Heartrate $-90 \% \operatorname{max~HR}-55 \% \max \mathrm{HR}$

Figure 15. Participant $n .7$ - curve of median values of heart rate in Zumba fitness

Notes: 58 - resting heart rate, 155- heart rate after warm up, 168 - average heart rate in main part, 140heart rate after final stretching.
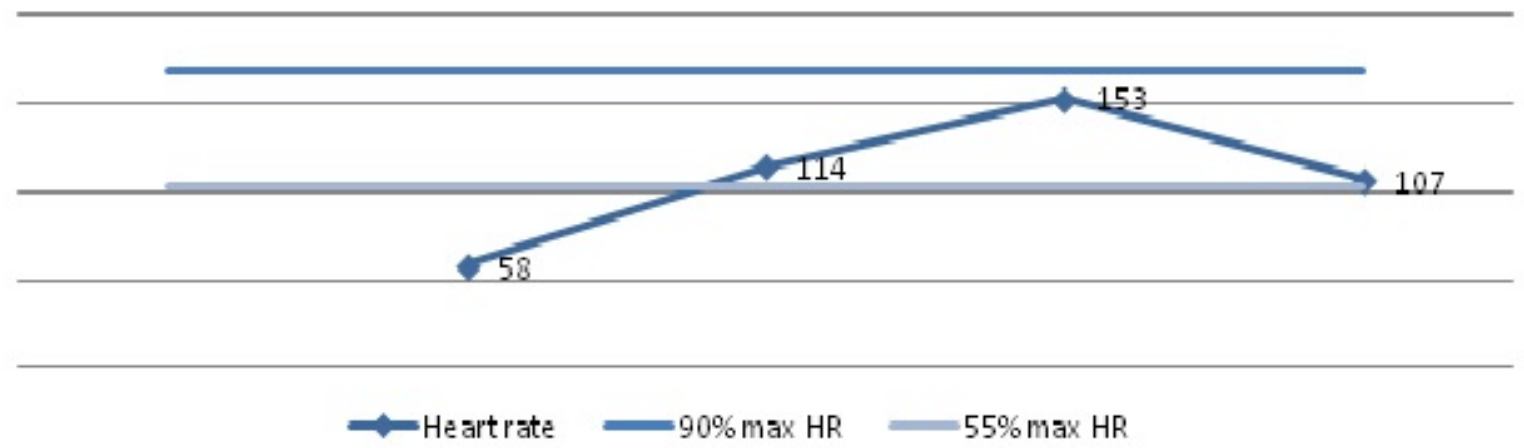

Figure 16. Participant n. 6 - curve of median values of heart rate in Tae-bo aerobics

Notes: 58 - resting heart rate, 114- heart rate after warm up, 153 - average heart rate in main part, 107heart rate after final stretching. 


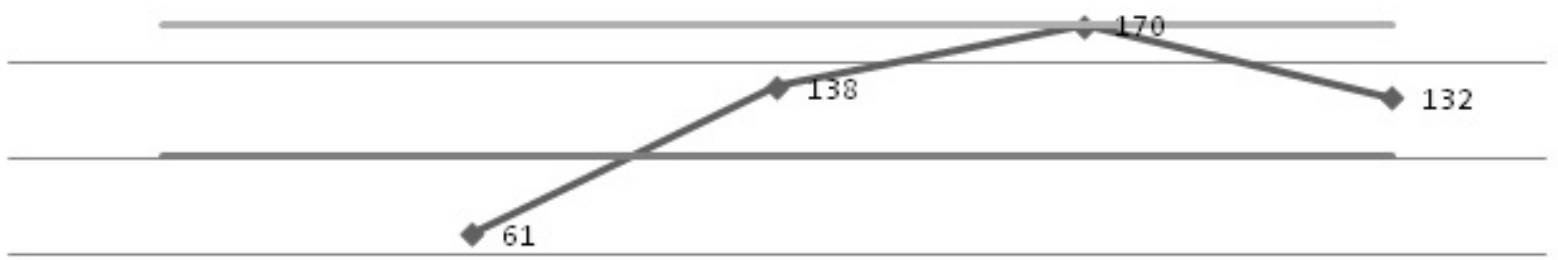

-Heartrate $-90 \% \max H R=55 \% \max H R$

Figure 17. Participant $n$. 8 - curve of median values of heart rate in Zumba fitness

Notes: 61 - resting heart rate, 138 - heart rate after warm up, 170 - average heart rate in main part, 132 heart rate after final stretching.

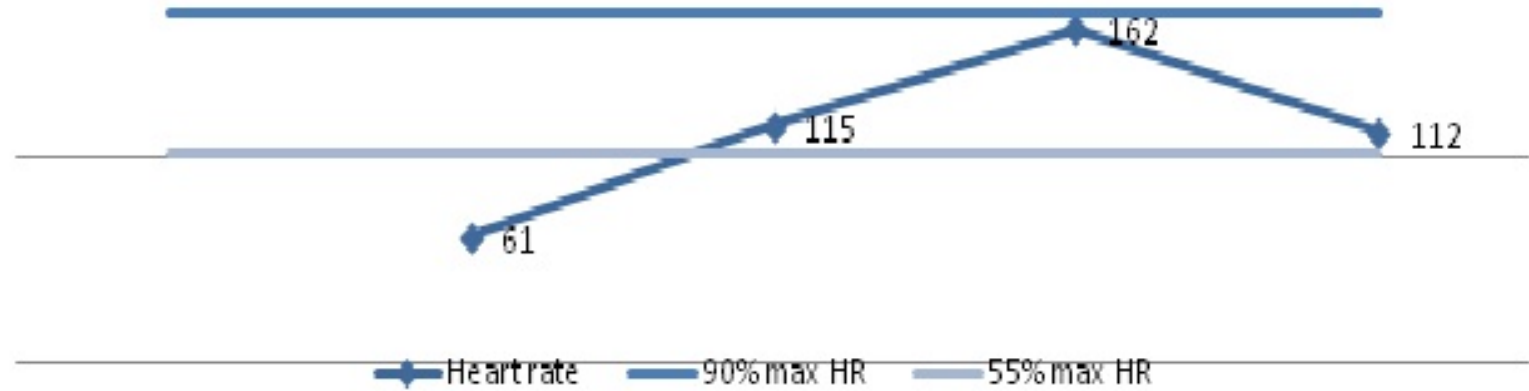

Figure 18. Participant n. 8 - curve of median values of heart rate in Tae-bo aerobics

Notes: 61 - resting heart rate, 115 - heart rate after warm up, 162 - average heart rate in main part, 112 heart rate after final stretching.
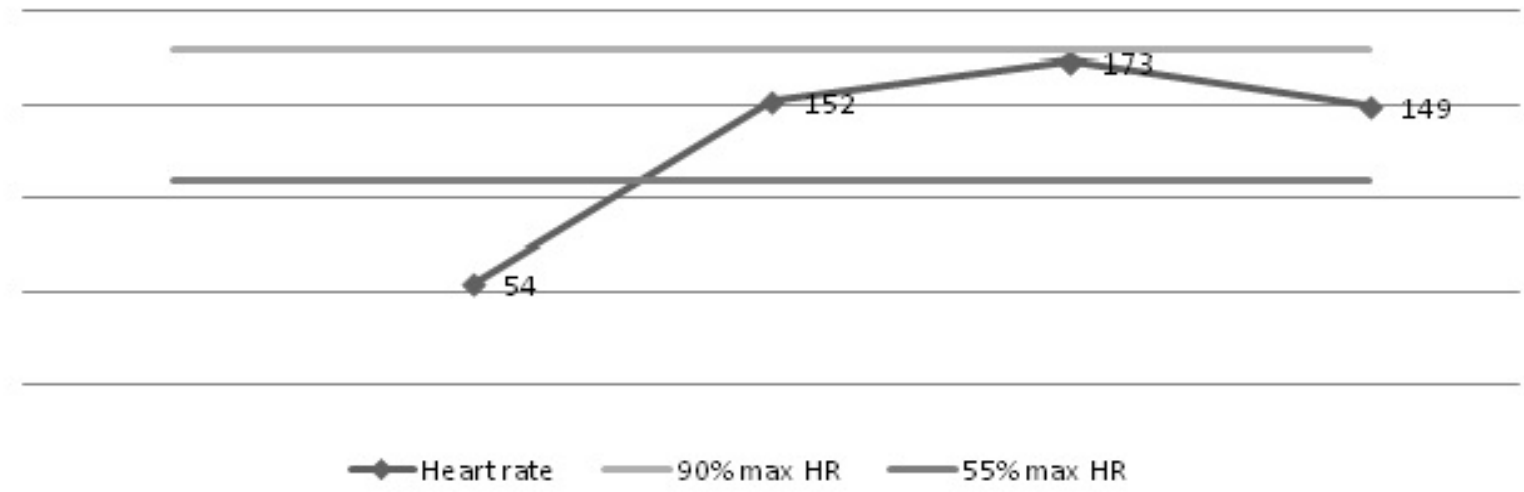

Figure 19. Participant $n .9$ - curve of median values of heart rate in Zumba fitness

Notes: 54 - resting heart rate, 152 - heart rate after warm up, 173 - average heart rate in main part, 149 heart rate after final stretching. 

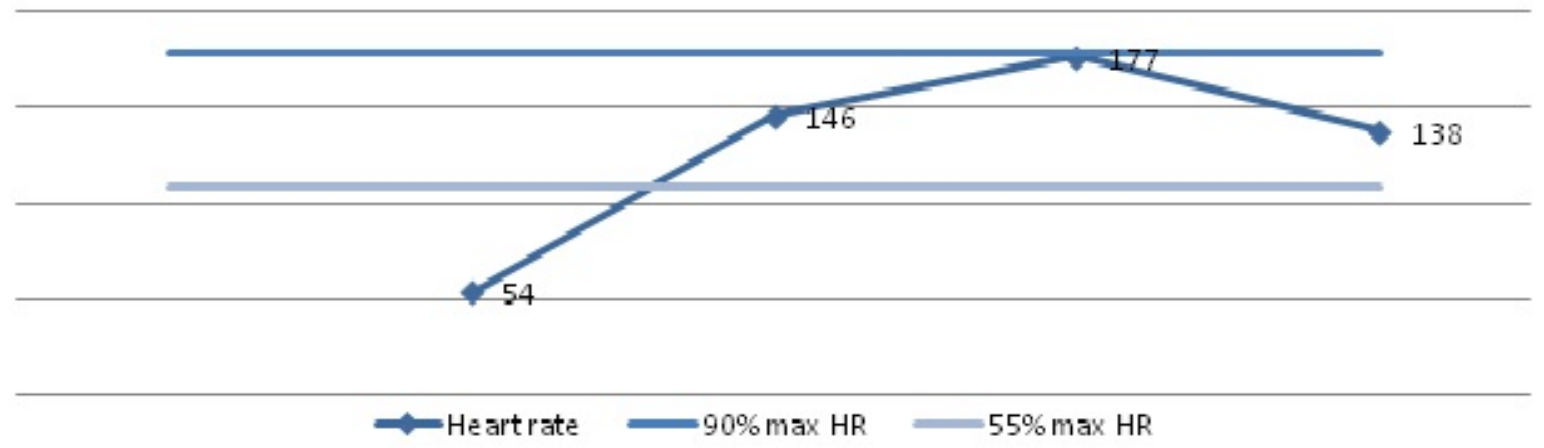

Figure 20. Participant n. 9 - curve of median values of heart rate in Tae-bo aerobics

Notes: 54 - resting heart rate, 146 - heart rate after warm up, 177 - average heart rate in main part, 138 heart rate after final stretching.

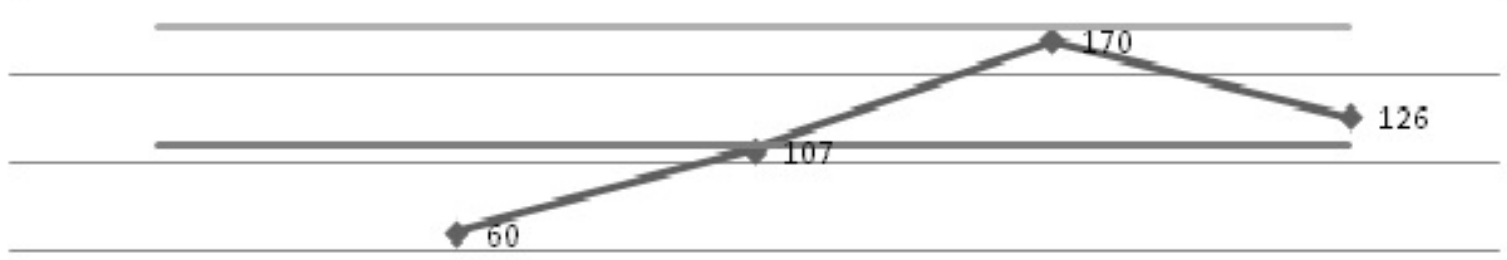

$\longrightarrow$ Heartrate $-90 \%$ max HR $-55 \%$ max HR

Figure 21. Participant $n .10$ - curve of median values of heart rate in Zumba fitness

Notes: 60 - resting heart rate, 107 - heart rate after warm up, 170 - average heart rate in main part, 126 heart rate after final stretching.
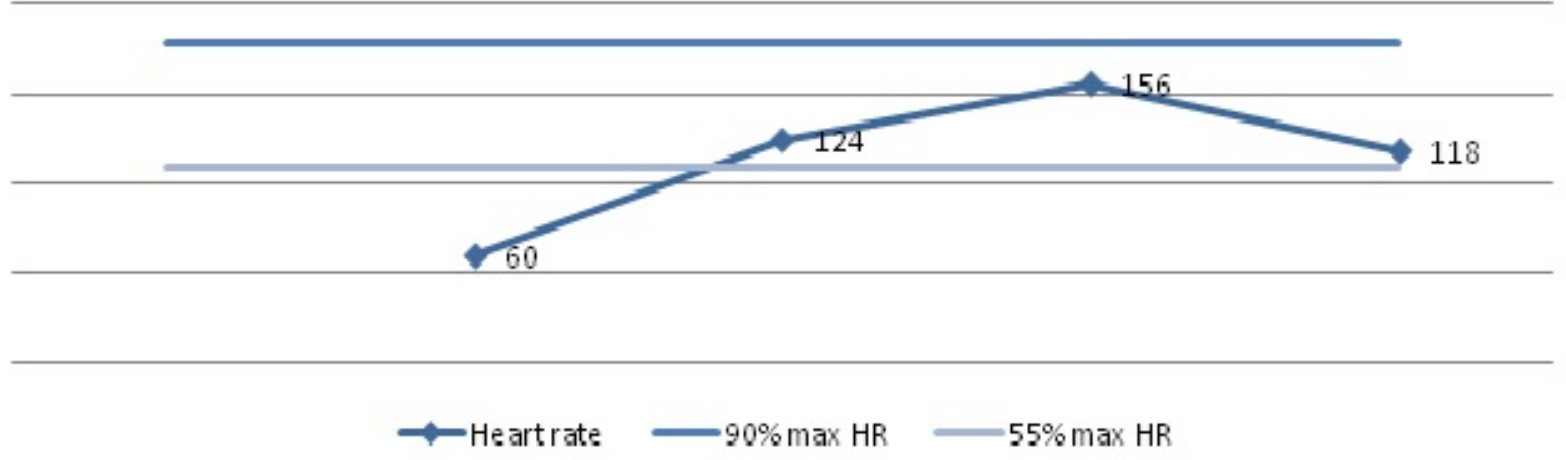

Figure 22. Participant $n .10$ - curve of median values of heart rate in Tae-bo aerobics

Notes: 60 - resting heart rate, 124 - heart rate after warm up, 156 - average heart rate in main part, 118 heart rate after final stretching. 

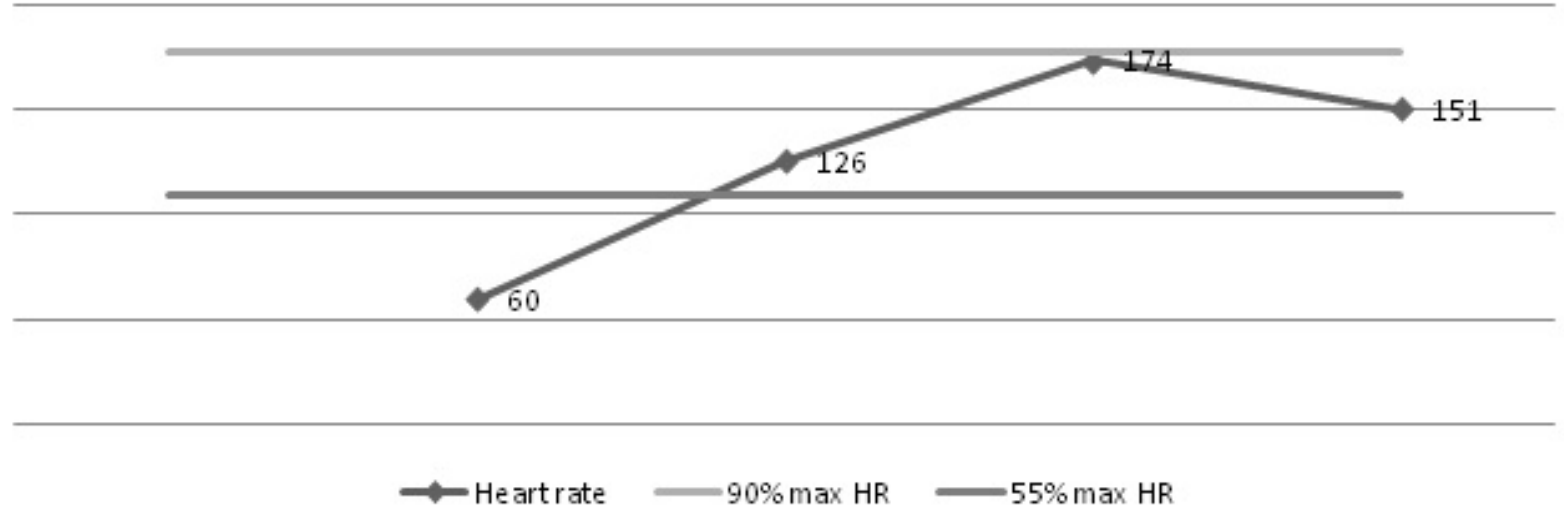

Figure 23. Participant $n .11$ - curve of median values of heart rate in Zumba fitness

Notes: 60 - resting heart rate, 126 - heart rate after warm up, 174 - average heart rate in main part, 151 heart rate after final stretching.
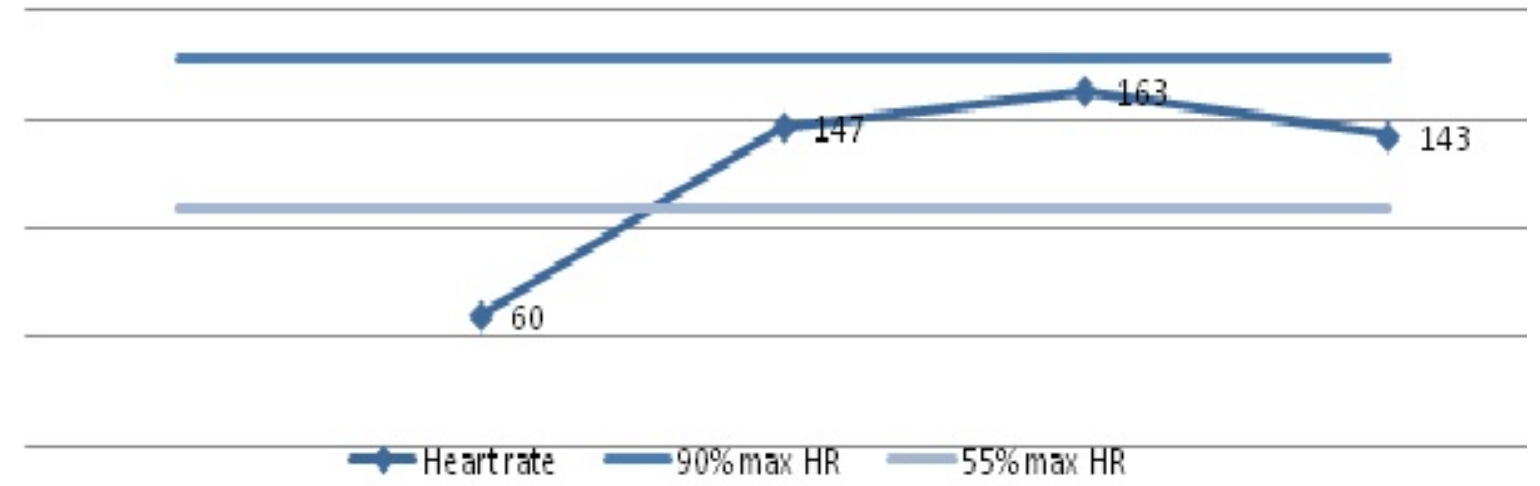

Figure 24. Participant $n .11$ - curve of median values of heart rate in Tae-bo aerobics

Notes: 60 - resting heart rate, 147 - heart rate after warm up, 163 - average heart rate in main part, 143 heart rate after final stretching.

Evaluating and comparing the measured values of heart rate after the warm ups in Zumba fitness lessons and Tae-bo aerobics lessons, we concluded that the values of heart rate in 8 tested women were higher after warming up in class Zumba fitness. Calculation according to Wilcoxon T-test, we found that the differences were statistically significant at the $5 \%$ level of significance (Figure. 25). 


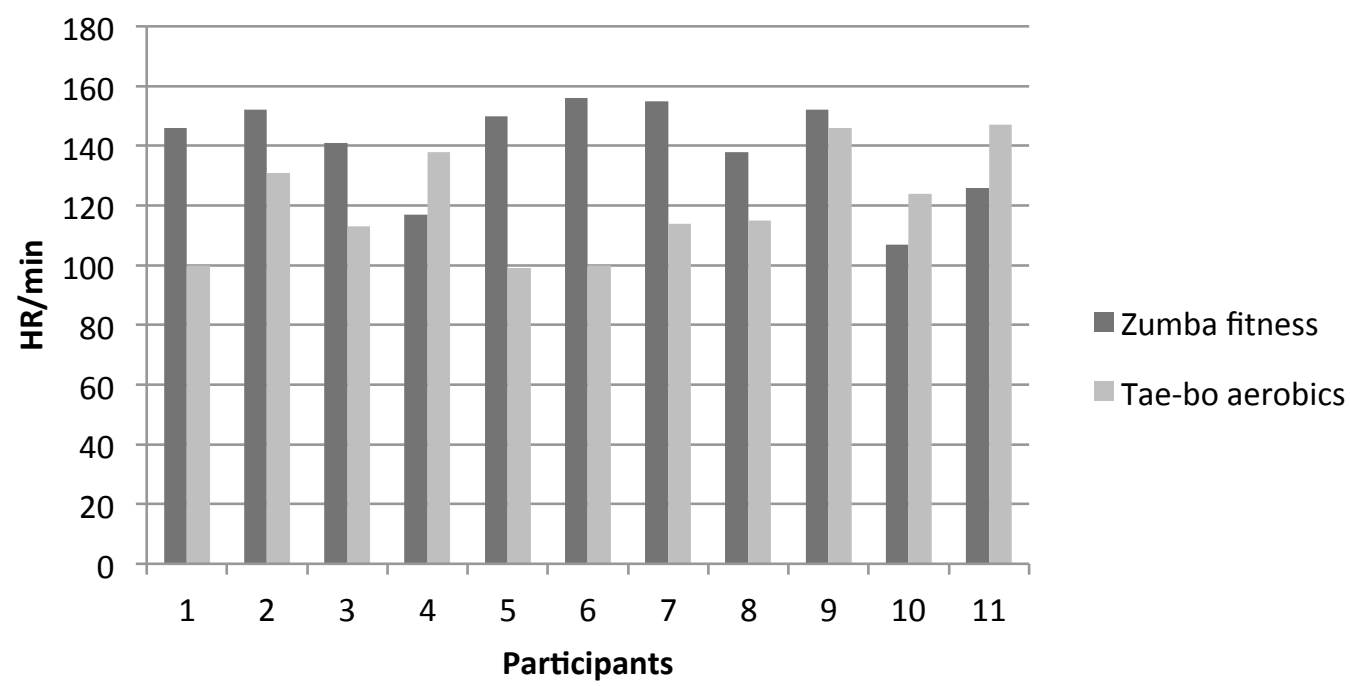

Figure 25. Median heart rate after warm ups

Differences in the values of heart rate in main parts in Zumba fitness classes and Tae-bo aerobics classes were statistically significant at the $5 \%$ level of significance (Figure. 26).

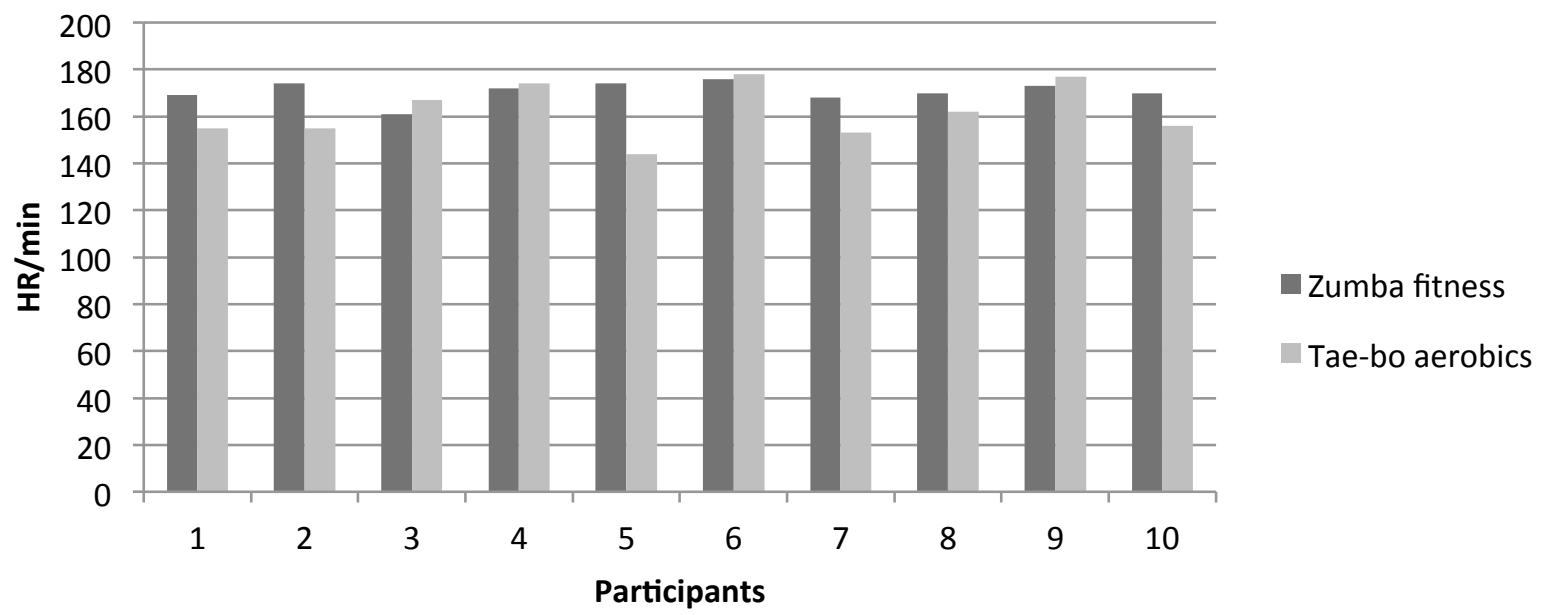

Figure 26. Median heart rate in main parts

Differences in heart rate values after the final stretch in Zumba fitness and Tae-bo aerobics classes were not statistically significant (Figure 27) 


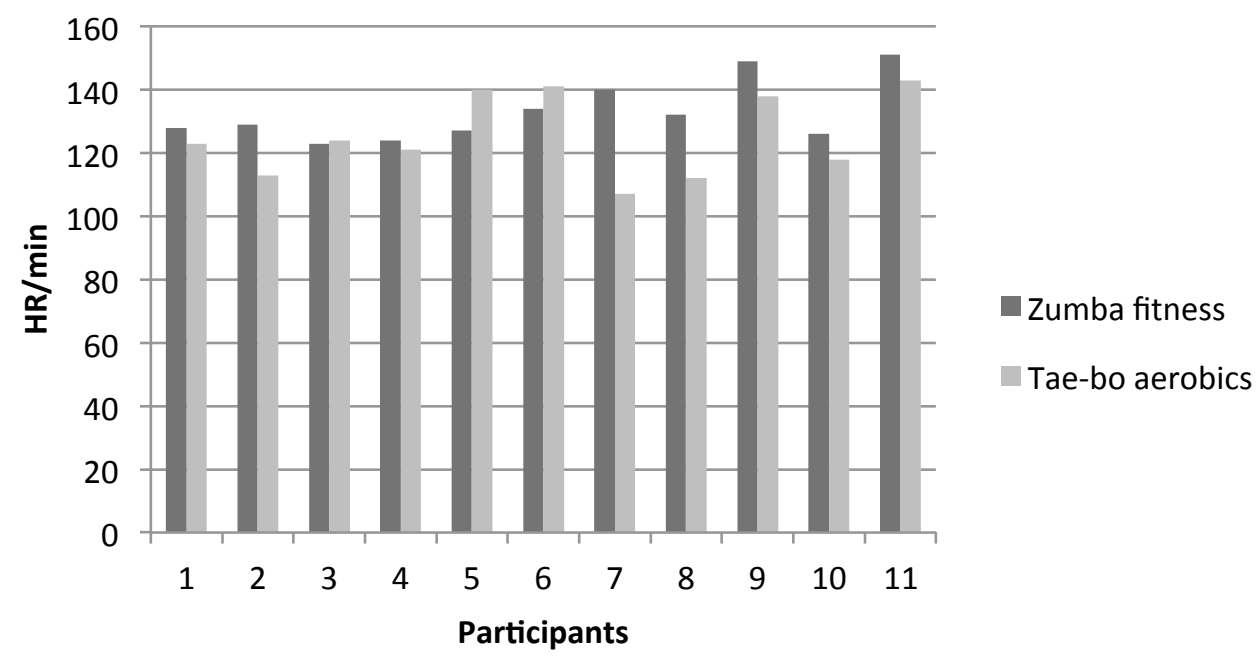

Figure 27. Median heart rate after final stretch

Differences in the values of energy consumption in Zumba fitness classes and Tae-bo aerobics classes are statistically significant at the $5 \%$ level of significance. The average value of energy consumption for 60 minutes Zumba fitness was $441 \mathrm{kcal}$. After the 60 minutes of Tae-bo aerobics an average value of energy consumption was $407 \mathrm{kcal}$ (Figure 28).

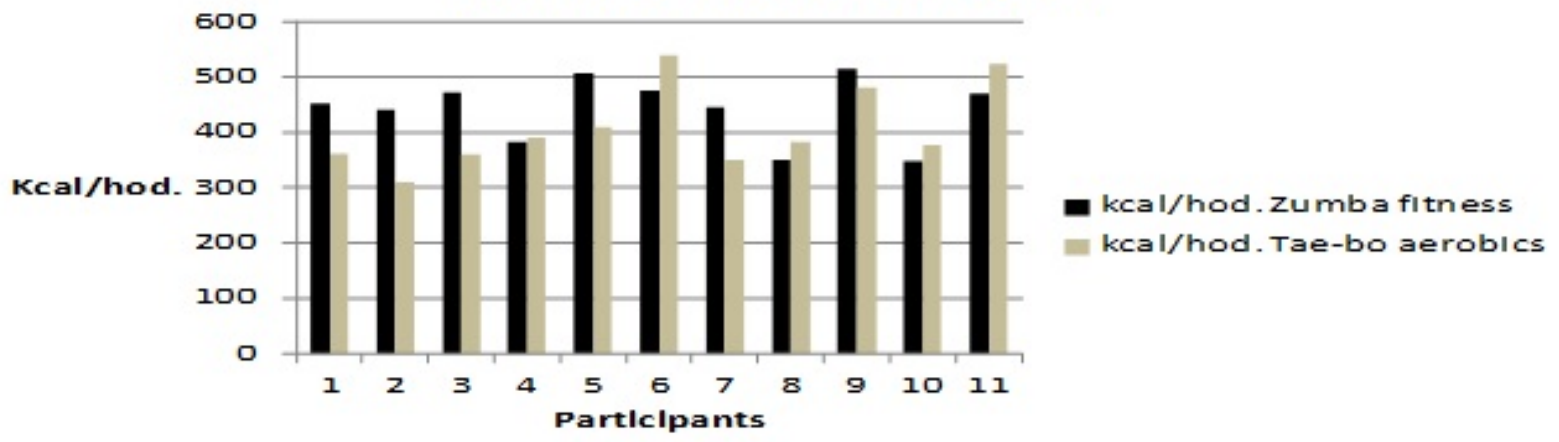

Figure 28. Median energy consumption

Basic statistics of the 66 measurements per Zumba fitness classes and 66 measurements per Tae-bo aerobics classes are shown in Tables 2 and 3.

Table 2. Basic statistics for Zumba fitness classes

\begin{tabular}{cccc}
\hline & Warm-up (HR) & Main part (HR) & Final (HR) \\
\hline Average & 139.2576 & 170.7121 & 132.3333 \\
Stdev & 15.23893 & 6.070775 & 9.935691 \\
Median & 143.5 & 170 & 130 \\
Min & 105 & 155 & 115 \\
Max & 158 & 182 & 155 \\
Variance & 53 & 27 & 35 \\
\hline
\end{tabular}


Table 3. Basic statistics for Tae-bo aerobics classes

\begin{tabular}{cccc}
\hline & Warm-up (HR) & Main part (HR) & Final (HR) \\
\hline Average & 120.7231 & 162.2344 & 125.7447 \\
Stdev & 16.52697 & 11.93068 & 13.64509 \\
Median & 120 & 161.5 & 123 \\
Min & 92 & 136 & 95 \\
Max & 151 & 186 & 158 \\
Variance & 59 & 50 & 63 \\
\hline
\end{tabular}

\section{DISCUSSION AND CONCLUSIONS}

Measurements have confirmed that the exercise intensity in the main parts of Zumba fitness was at 10 of the 11 participants in range $80 \%$ to $90 \%$ of maximum heart rate. In the main parts of tae-bo aerobics the exercise intensity was in range $80 \%$ to $90 \%$ of maximum heart rate at 8 of the 11 participants. Energy consumption in calories and exercise intensity were significantly higher in Zumba Fitness. The average value of energy consumption for 60 minutes of Zumba fitness workout was higher about $34 \mathrm{kcal}$ than of Tae-bo aerobics workout for the same time. It can be caused by power block in Tae-bo aerobics, while in Zumba fitness this block is missing. The standard deviation and variance of measurements were in Tea-bo aerobics higher, than in the Zumba fitness, so the intensity of the exercise varied in a larger range in Taebo aerobics. Again, this may be caused by weight training exercises.

\section{REFERENCES}

1. GREER N. Tae Bo: Fitness Craze or Effective speed. Aerobic and anaerobic exercises lead to a Workout? Vanderbilt University, Tennessee, U.S.A. significant improvement in the motor speed of the players http://www.vanderbilt.edu/AnS/psychology/health and that speed plays an important role in the sports when_psychology/TAEBO.htm. 2000.

2. HRČKA J. Šport pre všetkých- tvorba športovo- rekreačných programov. Bratislava: Manacon, 2000.

3. PEREZ B, GREENWOOD-ROBINSON M. Zumba. USA: Hachete Book Group, 2009. 